\title{
Distributed Fusion Estimation for Multisensor Multirate Systems with Stochastic Observation Multiplicative Noises
}

\author{
Peng Fangfang and Sun Shuli \\ School of Electronics Engineering, Heilongjiang University, Harbin 150080, China \\ Correspondence should be addressed to Sun Shuli; sunsl@hlju.edu.cn
}

Received 27 August 2013; Accepted 29 December 2013; Published 12 February 2014

Academic Editor: Wendong Xiao

Copyright (C) 2014 P. Fangfang and S. Shuli. This is an open access article distributed under the Creative Commons Attribution License, which permits unrestricted use, distribution, and reproduction in any medium, provided the original work is properly cited.

\begin{abstract}
This paper studies the fusion estimation problem of a class of multisensor multirate systems with observation multiplicative noises. The dynamic system is sampled uniformly. Sampling period of each sensor is uniform and the integer multiple of the state update period. Moreover, different sensors have the different sampling rates and observations of sensors are subject to the stochastic uncertainties of multiplicative noises. At first, local filters at the observation sampling points are obtained based on the observations of each sensor. Further, local estimators at the state update points are obtained by predictions of local filters at the observation sampling points. They have the reduced computational cost and a good real-time property. Then, the cross-covariance matrices between any two local estimators are derived at the state update points. At last, using the matrix weighted optimal fusion estimation algorithm in the linear minimum variance sense, the distributed optimal fusion estimator is obtained based on the local estimators and the cross-covariance matrices. An example shows the effectiveness of the proposed algorithms.
\end{abstract}

\section{Introduction}

In networked systems or sensor networks, there often exist various uncertainties during the transmission process of signals due to the imperfection of the communication channels. It makes impossible to use linear model to describe some systems. The uncertainties can be approximated mathematically by an additive noise or a multiplicative noise [1-6]. These systems are widely used in petroleum seismic exploration, target detection, speech processing, and other areas; thus, the research on systems with multiplicative noise has the important practical significance. In the early references [1], the optimal linear filters have been proposed for systems with uncertain observations described by the multiplicative noise. For more general case with stochastic parameters, the optimal linear estimation is designed in [2]. References [3-5] study the polynomial filters; however, the proposed nonlinear filters have expensive computational cost. For networked systems with multiplicative noises and packet dropouts, optimal linear estimators including filter, predictor, and smoother have been proposed in [6]. However, the above-mentioned literatures are all concerned with single sensor case but do not take multiple sensors into account.

As the sensor technology is widely used in military, civilian, scientific research, and many other fields, single sensor has failed to meet the performance requirements in many aspects. Moreover, as the development of electronics technologies, various sensors have been developed and applied to many practical fields such as target tracking since they can provide more information than any single sensor. Therefore, multisensor information fusion has received considerable research attention in recent years [7]. For systems with a single sampling rate, the optimal state weighted fusion filter in the linear minimum variance sense [8] and the self-tuning fusion filter with unknown noise variances [9] have been presented. Recently, the multirate multisensor asynchronous fusion algorithms have been studied in [10-12]. References $[13,14]$ adopt the state augmentation approach to give the estimators with the expensive computational cost. Though $[15,16]$ adopt the nonaugmented approach to design the filters, a modeling error is made by ignoring the process noise. Therefore, there is the accuracy loss. By considering 


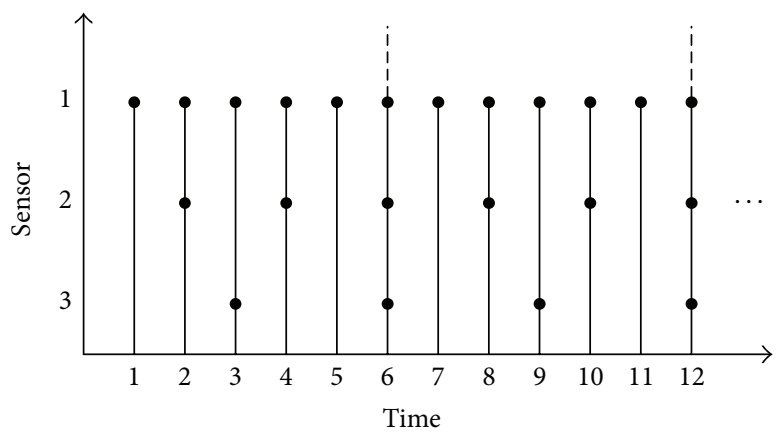

FIGURE 1: Sampling case of sensors.

the process noise to eliminate the modeling error, an optimal filter is presented to improve the estimation accuracy [17]. Furthermore, the missing measurements are also taken into account in [16, 18]. In [19], a multirate multisensor distributed fusion estimator is proposed for two-sensor systems with one-step cross-covariance noises. However, most of the above-mentioned literatures do not take the multiplicative noises into account. In sensor networks, there often exist various sensors with different sampling rates and stochastic uncertainty of multiplicative noises. It is significant to use the nonaugmented approach to deal with the multirate multisensor systems. This motivates our work.

This paper studies the fusion estimation problem of a class of multisensor multirate systems with observation multiplicative noises. State is sampled uniformly at the finest rate. Different sensors have different sampling periods that are integer multiples of the state update period. Local estimators at the state sampling points are obtained based on the local filters at the observation sampling points by the filtering and prediction. By using the distributed optimal weighted fusion estimation algorithm in the linear minimum variance sense [8], a distributed optimal fusion estimator is obtained. It avoids the state and observation augmentation. It has a good reliability since it has the distributed parallel structure. Moreover, the estimation error cross-covariance matrices between any two local estimators are derived according to the different sampling cases.

\section{Problem Formulation}

Consider the following multisensor multirate system with observation multiplicative noises:

$$
\begin{gathered}
x(t+1)=\Phi x(t)+\Gamma w(t), \\
y_{i}\left(l_{i} t\right)=\left(H_{0 i}+\xi_{i}\left(l_{i} t\right) H_{1 i}\right) x\left(l_{i} t\right)+v_{i}\left(l_{i} t\right), \\
i=1,2, \ldots L,
\end{gathered}
$$

where $x(t)$ is the system state at time $t T$ and $T$ is the state update period. $\Phi, \Gamma, H_{0 i}$, and $H_{1 i}$ are constant matrices. $y_{i}\left(l_{i} t\right)$ is the observation of the $i$ th sensor at time $l_{i} t T$; $l_{i}$ is the ratio of the observation sampling period and the state update period. $L$ is the number of sensors. $w(t)$ and $v_{i}\left(l_{i} t\right)$ are white noises with zero mean and variances $Q_{w}$ and $Q_{v_{i}}$, respectively.
The observation multiplicative noise $\xi_{i}\left(l_{i} t\right)$ is scalar white noise with zero mean and variance $Q_{\xi_{i}} \cdot w(t), v_{i}\left(l_{i} t\right)$, and $\xi_{i}\left(l_{i} t\right)$ are uncorrelated with each other. The initial state $x(0)$ is independent of $w(t), v_{i}\left(l_{i} t\right)$, and $\xi_{i}\left(l_{i} t\right)$ and satisfies that $E\{x(0)\}=\mu_{0}$ and $E\left\{\left[x(0)-\mu_{0}\right]\left[x(0)-\mu_{0}\right]^{T}\right\}=P_{0}$, where the symbol $E$ is the mathematical expectation.

Remark 1. The sampling case of multisensor multirate systems can be described by Figure 1. The horizontal axis denotes time while the vertical axis denotes different sensors. Three sensors are shown in Figure 1. Black circle solid points represent the sampling time of different sensors. The sampling rate goes from the highest (sensor 1) to the lowest (sensor 3). As shown in Figure 1, the three sensors all sample uniformly. The first sensor has the same sampling rate as the state update rate; that is, the sampling period is $T$. The sampling period of the second sensor is $2 T$ and the third is $3 T$. It is clear that the least common multiple of three sample periods is $6 T$. This means that the samplings of different sensors are asynchronous in each data block of the length $6 T$.

The objective of this paper is to find the distributed optimal fusion estimator $\widehat{x}_{o}(t)$ of $x(t)$ based on the local estimators $\widehat{x}_{i}(t)$ from different sensors.

To obtain the distributed fusion estimator by using the optimal weighted fusion estimation algorithm in the linear minimum variance sense [8], we need to compute the local estimators and variance matrices from each sensor and the cross-covariance matrices between any two local estimators. In the latter text, we will give the computation of local estimators and cross-covariance matrices.

\section{Local Filters at the Observation Sampling Points}

At first, we give the filter at the observation sampling points of each sensor.

From the iteration of (1), we have

$$
\begin{aligned}
x\left(l_{i} t+l_{i}\right)= & \Phi^{l_{i}} x\left(l_{i} t\right) \\
& +\sum_{m=0}^{l_{i}-1} \Phi^{m} \Gamma w\left(l_{i} t+l_{i}-m-1\right) .
\end{aligned}
$$


Let $\Phi_{i}=\Phi^{l_{i}}$ and $w_{i}\left(l_{i} t\right)=\sum_{m=0}^{l_{i}-1} \Phi^{m} \Gamma w\left(l_{i} t+l_{i}-m-1\right)$; we have the state space model at the observation sampling points for the $i$ th sensor as follows:

$$
\begin{array}{r}
x\left(l_{i} t+l_{i}\right)=\Phi_{i} x\left(l_{i} t\right)+w_{i}\left(l_{i} t\right), \\
y_{i}\left(l_{i} t\right)=\left(H_{0 i}+\xi_{i}\left(l_{i} t\right) H_{1 i}\right) x\left(l_{i} t\right)+v_{i}\left(l_{i} t\right), \\
i=1,2, \ldots, L
\end{array}
$$

with the noise statistical information $Q_{w_{i}}=E\left\{w_{i}\left(l_{i} t\right)\right.$ $\left.w_{i}\left(l_{i} t\right)^{T}\right\}=\sum_{m=0}^{l_{i}-1} \Phi^{m} \Gamma Q_{w} \Gamma^{T}\left(\Phi^{m}\right)^{T}$.

Then, we have the filter at the observation sampling points of each sensor based on the above model.

Lemma 2 (see [6]). For system (4), the local filters at the observation sampling points of the ith sensor are computed by

$$
\begin{gathered}
\widehat{x}_{i}\left(l_{i} t \mid l_{i} t\right)=\widehat{x}_{i}\left(l_{i} t \mid l_{i} t-l_{i}\right)+K_{i}\left(l_{i} t\right) \varepsilon_{i}\left(l_{i} t\right), \\
\widehat{x}_{i}\left(l_{i} t+l_{i} \mid l_{i} t\right)=\Phi_{i} \widehat{x}_{i}\left(l_{i} t \mid l_{i} t\right), \\
\varepsilon_{i}\left(l_{i} t\right)=y_{i}\left(l_{i} t\right)-H_{0 i} \widehat{x}_{i}\left(l_{i} t \mid l_{i} t-l_{i}\right), \\
K_{i}\left(l_{i} t\right)=P_{i}\left(l_{i} t \mid l_{i} t-l_{i}\right) H_{0 i}^{T} Q_{\varepsilon i}^{-1}\left(l_{i} t\right), \\
Q_{\varepsilon i}\left(l_{i} t\right)=H_{0 i} P_{i}\left(l_{i} t \mid l_{i} t-l_{i}\right) H_{0 i}^{T} \\
\quad+H_{1 i} Q_{\xi_{i}} q_{i}\left(l_{i} t\right) H_{1 i}^{T}+Q_{v_{i}}, \\
P_{i}\left(l_{i} t \mid l_{i} t\right)=\left(I_{n}-K_{i}\left(l_{i} t\right) H_{0 i}\right) P_{i}\left(l_{i} t \mid l_{i} t-l_{i}\right), \\
P_{i}\left(l_{i} t+l_{i} \mid l_{i} t\right)=\Phi_{i} P_{i}\left(l_{i} t \mid l_{i} t\right) \Phi_{i}^{T}+Q_{w_{i}}, \\
q_{i}\left(l_{i} t\right)=\Phi_{i} q_{i}\left(l_{i} t-l_{i}\right) \Phi_{i}^{T}+Q_{w_{i}},
\end{gathered}
$$

where $\widehat{x}_{i}\left(l_{i} t \mid l_{i} t\right)$ and $\hat{x}_{i}\left(l_{i} t \mid l_{i} t-l_{i}\right)$ are the filter and predictor at the observation sampling points, respectively. $P_{i}\left(l_{i} t \mid l_{i} t\right)$ and $P_{i}\left(l_{i} t \mid l_{i} t-l_{i}\right)$ are the corresponding covariance matrices. $\varepsilon_{i}\left(l_{i} t\right)$ is the innovation sequence with the covariance matrix $Q_{\varepsilon i}\left(l_{i} t\right)$. $K_{i}\left(l_{i} t\right)$ is the filtering gain matrix. $q_{i}\left(l_{i} t\right)$ is the state secondorder moment. The initial values are $\widehat{x}_{i}(0 \mid 0)=\mu_{0}, P_{i}(0 \mid 0)=$ $P_{0}$, and $q_{i}(0)=\mu_{0} \mu_{0}^{T}+P_{0}$.

\section{Local Estimators at the State Update Points}

Based on the filters at the observation sampling points in Lemma 2, we have the following state estimation algorithms at the state update points.

Theorem 3. For system (1)-(2), the local estimators at the state update points of the ith sensor are computed by

$$
\widehat{x}_{i}(t)= \begin{cases}\widehat{x}_{i}\left(l_{i} l \mid l_{i} l\right), & t=l_{i} l, l=0,1,2, \ldots \\ \Phi^{p} \widehat{x}_{i}\left(l_{i} l \mid l_{i} l\right), & t=l_{i} l+p, p=1,2, \ldots, l_{i}-1 .\end{cases}
$$

The estimation error covariance matrices are computed by

$$
\begin{aligned}
& P_{i}(t) \\
& = \begin{cases}P_{i}\left(l_{i} l \mid l_{i} l\right), & t=l_{i} l, l=0,1,2, \ldots \\
\Phi^{p} P_{i}\left(l_{i} l \mid l_{i} l\right)\left(\Phi^{p}\right)^{T} & \\
\quad+\sum_{m=0}^{p-1} \Phi^{m} \Gamma Q_{w} \Gamma^{T}\left(\Phi^{m}\right)^{T}, & t=l_{i} l+p, p=1,2, \ldots, l_{i}-1,\end{cases}
\end{aligned}
$$

where $\widehat{x}_{i}\left(l_{i} l \mid l_{i} l\right)$ and $P_{i}\left(l_{i} l \mid l_{i} l\right)$ are computed by Lemma 2 .

Proof. When $t=l_{i} l, l=0,1,2, \ldots$, we have the filters $\widehat{x}_{i}(t)=$ $\widehat{x}_{i}\left(l_{i} l \mid l_{i} l\right)$. When $t=l_{i} l+p, p=1,2, \ldots, l_{i}-1$, we have the predictors $\widehat{x}_{i}(t)=\widehat{x}_{i}\left(l_{i} l+p \mid l_{i} l\right)$. Then from the iteration of (1), we have

$$
\begin{aligned}
x\left(l_{i} l+p\right)= & \Phi^{p} x\left(l_{i} l\right) \\
& +\sum_{m=0}^{p-1} \Phi^{m} \Gamma w\left(l_{i} l+p-m-1\right), \\
& \quad p=1,2, \ldots l_{i}-1 .
\end{aligned}
$$

Taking projection of both sides of (8) onto the linear space $\left\{y_{i}(0), y_{i}\left(l_{i}\right), \ldots, y_{i}\left(l_{i} l\right)\right\}$, we have the second equation of $(6)$.

From (6) and (8), we easily obtain the estimation error equations:

$$
\begin{aligned}
& \widetilde{x}_{i}(t)
\end{aligned}
$$

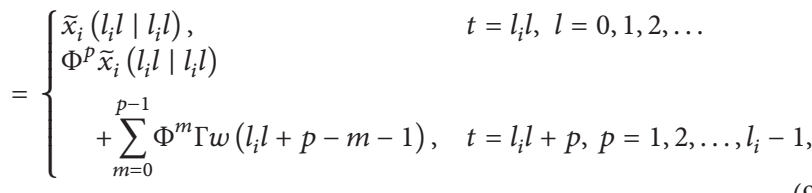

where the estimation error is $\widetilde{x}_{i}(t)=x(t)-\widehat{x}_{i}(t)$. Substituting (9) into $P_{i}(t)=E\left[\tilde{x}_{i}(t) \tilde{x}_{i}^{T}(t)\right]$, we have (7). This proof is completed.

Remark 4. The local estimators at the state update points have been obtained by filtering and prediction based on the filter at the observation sampling points. State augmentation is avoided. They are simple and have a good real-time property.

Now, we have obtained the local estimators at the state update points based on the observations of each sensor. Next, we compute the cross-covariance matrices between any two local estimators.

\section{Computation of Cross-Covariance Matrix}

Theorem 5. The estimation error cross-covariance matrices between any two local estimators can be computed in the following three cases. 
(i) If the ith sensor and the jth sensor both have observations at time $t T$, the estimation error cross-covariance matrix is given as

$$
\begin{aligned}
P_{i j}(t)= & \Phi P_{i j}(t-1) \Phi^{T}+\Gamma Q_{w} \Gamma^{T} \\
& +K_{i}(t) H_{0 i}\left(\Phi P_{i j}(t-1) \Phi^{T}+\Gamma Q_{w} \Gamma^{T}\right) H_{0 j}^{T} K_{j}^{T}(t) \\
& -\Phi P_{i j}(t-1) \Phi^{T} H_{0 j}^{T} K_{j}^{T}(t)-\Gamma Q_{w} \Gamma^{T} H_{0 j}^{T} K_{j}^{T}(t) \\
& -K_{i}(t) H_{0 i} \Phi P_{i j}(t-1) \Phi^{T}-K_{i}(t) H_{0 i} \Gamma Q_{w} \Gamma^{T} .
\end{aligned}
$$

(ii) If the ith sensor has an observation and the jth sensor does not have any observation at time $t T$, the estimation error cross-covariance matrix is given as

$$
\begin{aligned}
P_{i j}(t)= & \Phi P_{i j}(t-1) \Phi^{T}+\Gamma Q_{w} \Gamma^{T} \\
& -K_{i}(t) H_{0 i} \Phi P_{i j}(t-1) \Phi^{T}-K_{i}(t) H_{0 i} \Gamma Q_{w} \Gamma^{T}
\end{aligned}
$$

(iii) If both the ith sensor and the jth sensor do not have observations at time $t T$, the estimation error cross-covariance matrix is given as

$$
P_{i j}(t)=\Phi P_{i j}(t-1) \Phi^{T}+\Gamma Q_{w} \Gamma^{T}
$$

The initial value is $P_{i j}(0)=P_{0}$.

Proof. (i) If the $i$ th sensor and the $j$ th sensor both have observations at time $t T$, we have local filters as

$$
\begin{aligned}
\widehat{x}_{i}(t) & =\widehat{x}_{i}(t \mid t-1)+K_{i}(t) \varepsilon_{i}(t) \\
& =\Phi \widehat{x}_{i}(t-1)+K_{i}(t) \varepsilon_{i}(t), \\
\widehat{x}_{j}(t) & =\widehat{x}_{j}(t \mid t-1)+K_{j}(t) \varepsilon_{j}(t) \\
& =\Phi \widehat{x}_{j}(t-1)+K_{j}(t) \varepsilon_{j}(t),
\end{aligned}
$$

where

$$
\begin{aligned}
& \widehat{x}_{i}(t-1)=\widehat{x}_{i}\left(t-1 \mid t-l_{i}\right), \\
& \widehat{x}_{j}(t-1)=\widehat{x}_{j}\left(t-1 \mid t-l_{j}\right) .
\end{aligned}
$$

Then we obtain the estimation error equation as

$$
\begin{aligned}
\tilde{x}_{i}(t)= & \Phi \tilde{x}_{i}\left(t-1 \mid t-l_{i}\right) \\
& +\Gamma w(t-1)-K_{i}(t) \varepsilon_{i}(t), \\
\tilde{x}_{j}(t)= & \Phi \tilde{x}_{j}\left(t-1 \mid t-l_{j}\right) \\
& +\Gamma w(t-1)-K_{j}(t) \varepsilon_{j}(t) .
\end{aligned}
$$

The cross-covariance matrix can be computed by

$$
\begin{aligned}
P_{i j}(t)=E & {\left[\tilde{x}_{i}(t \mid t) \tilde{x}_{j}^{T}(t \mid t)\right] } \\
=\Phi & P_{i j}(t-1) \Phi^{T}+\Gamma Q_{w} \Gamma^{T} \\
& +K_{i}(t) E\left\{\varepsilon_{i}(t) \varepsilon_{j}^{T}(t)\right\} K_{j}^{T}(t) \\
& -\Phi E\left\{\tilde{x}_{i}\left(t-1 \mid t-l_{j}\right) \varepsilon_{j}^{T}(t)\right\} K_{j}^{T}(t) \\
& -\Gamma E\left\{w(t-1) \varepsilon_{j}^{T}(t)\right\} K_{j}^{T}(t) \\
& -K_{i}(t) E\left\{\varepsilon_{i}(t) \tilde{x}_{j}^{T}\left(t-1 \mid t-l_{j}\right)\right\} \Phi^{T} \\
& -K_{i}(t) E\left\{\varepsilon_{i}(t) w^{T}(t-1)\right\} \Gamma^{T},
\end{aligned}
$$

where

$$
\begin{gathered}
E\left\{\varepsilon_{i}(t) \varepsilon_{j}^{T}(t)\right\} \\
=H_{0 i}\left(\Phi P_{i j}\left(t-1 \mid t-l_{i}, t-l_{j}\right) \Phi^{T}+\Gamma Q_{w} \Gamma^{T}\right) H_{0 j}^{T}, \\
E\left\{w(t-1) \varepsilon_{j}^{T}(t)\right\}=Q_{w} \Gamma^{T} H_{0 j}^{T}, \\
E\left\{\varepsilon_{i}(t) \tilde{x}_{j}^{T}\left(t-1 \mid t-l_{j}\right)\right\} \\
=H_{0 i} \Phi P_{i j}\left(t-1 \mid t-l_{i}, t-l_{j}\right) .
\end{gathered}
$$

Substituting (17) into (24) and noting that $P_{i j}(t-1)=$ $P_{i j}\left(t-1 \mid t-l_{i}, t-l_{j}\right),(10)$ is obtained.

(ii) If the $i$ th sensor has an observation and the $j$ th sensor does not have any observation at time $t T$, we have local estimators as

$$
\begin{aligned}
\widehat{x}_{i}(t) & =\widehat{x}_{i}(t \mid t-1)+K_{i}(t) \varepsilon_{i}(t) \\
& =\Phi \widehat{x}_{i}(t-1)+K_{i}(t) \varepsilon_{i}(t), \\
\widehat{x}_{j}(t) & =\widehat{x}_{j}(t \mid t-1)=\Phi \widehat{x}_{j}(t-1) .
\end{aligned}
$$

Then, we have the estimation error equation as

$$
\begin{aligned}
\tilde{x}_{i}(t)= & \Phi \tilde{x}_{i}\left(t-1 \mid t-l_{i}\right) \\
& +\Gamma w(t-1)-K_{i}(t) \varepsilon_{i}(t), \\
\tilde{x}_{j}(t)= & \tilde{x}_{j}(t \mid t-1) \\
= & \Phi \tilde{x}_{j}\left(t-1 \mid t-l_{j}\right)+\Gamma w(t-1) .
\end{aligned}
$$

Similarly to the derivation of the case (i), (11) can be obtained by computing $P_{i j}(t)=E\left[\tilde{x}_{i}(t) \tilde{x}_{j}^{T}(t)\right]$.

(iii) If both the $i$ th sensor and the $j$ th sensor do not have observations at time $t T$, we have local estimators as

$$
\begin{aligned}
\widehat{x}_{i}(t) & =\widehat{x}_{i}(t \mid t-1) \\
& =\Phi \widehat{x}_{i}(t-1)=\Phi \widehat{x}_{i}\left(t-1 \mid t-l_{i}\right), \\
\widehat{x}_{j}(t) & =\widehat{x}_{j}(t \mid t-1) \\
& =\Phi \widehat{x}_{j}(t-1)=\Phi \widehat{x}_{j}\left(t-1 \mid t-l_{j}\right) .
\end{aligned}
$$




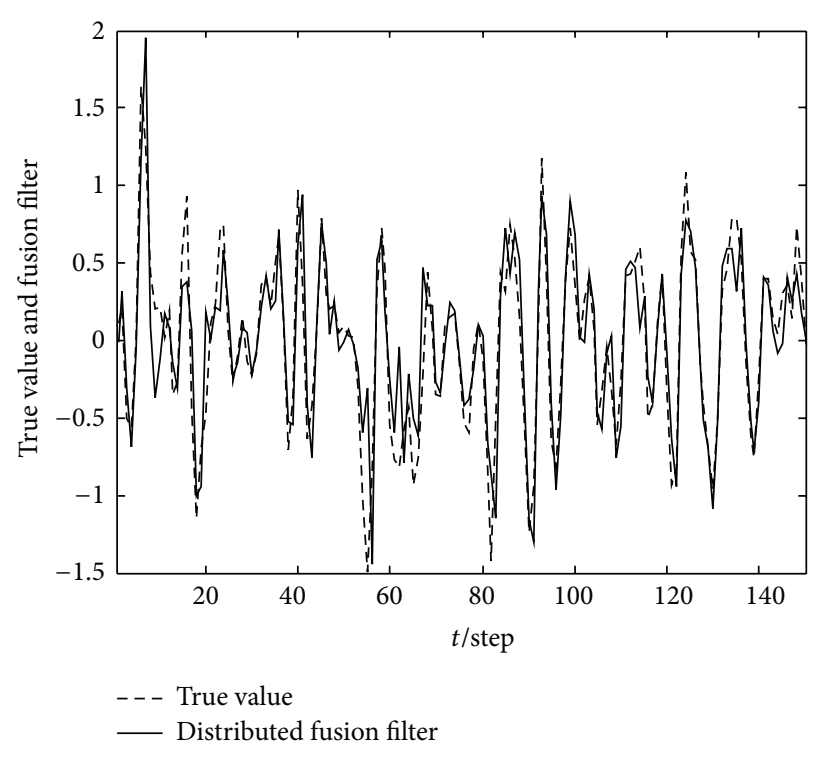

(a) The first state component

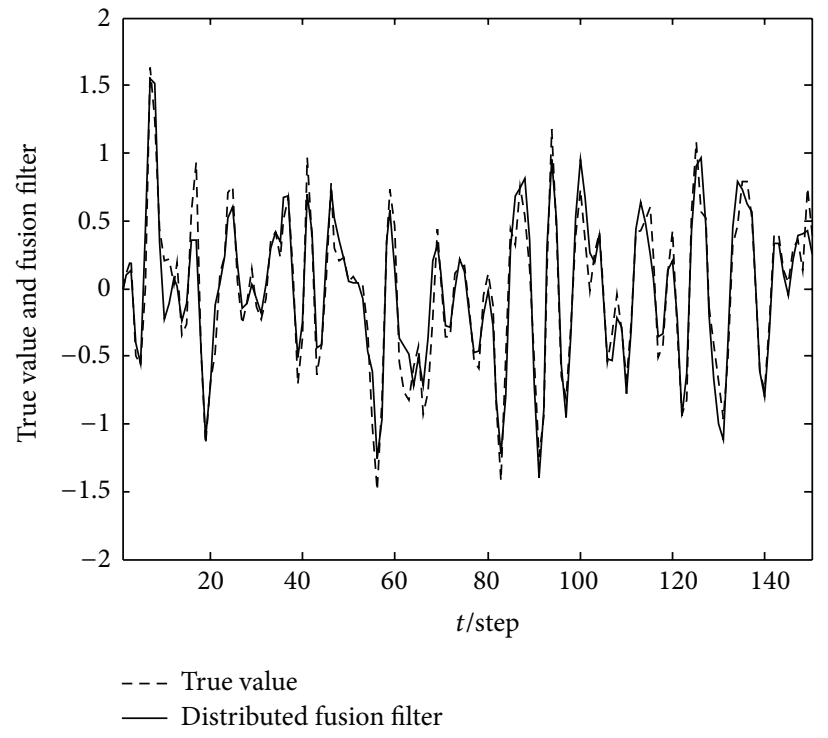

(b) The second state component

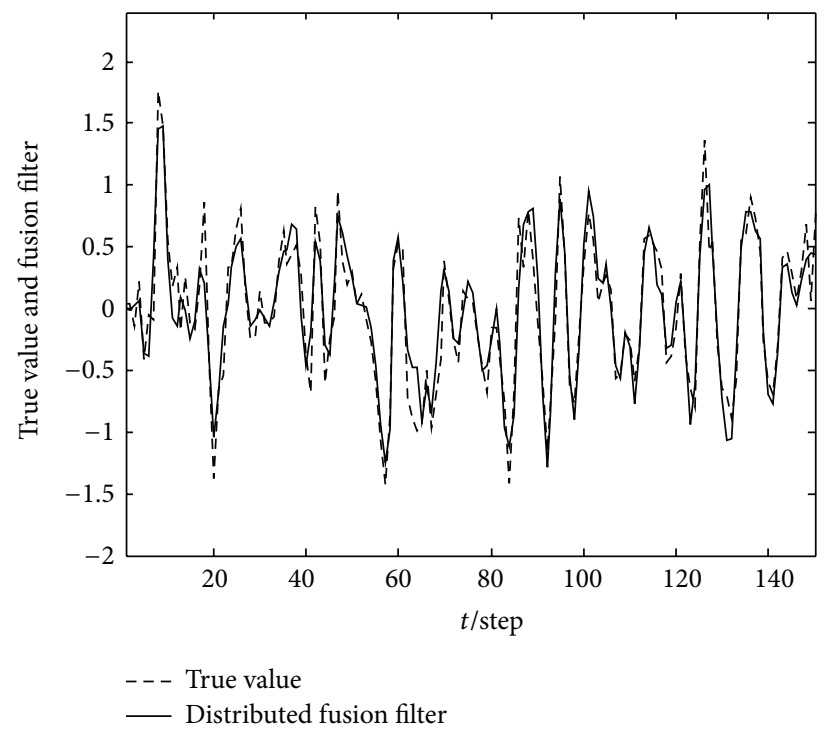

(c) The third state component

FIGURE 2: Distributed fusion filter.

We have the estimation error equation as

$$
\begin{aligned}
\tilde{x}_{i}(t) & =\tilde{x}_{i}(t \mid t-1) \\
& =\Phi \tilde{x}_{i}\left(t-1 \mid t-l_{i}\right)+\Gamma w(t-1), \\
\tilde{x}_{j}(t) & =\tilde{x}_{j}(t \mid t-1) \\
& =\Phi \tilde{x}_{j}\left(t-1 \mid t-l_{i}\right)+\Gamma w(t-1) .
\end{aligned}
$$

Then (12) is obtained by computing $P_{i j}(t)=E\left[\tilde{x}_{i}(t) \tilde{x}_{j}^{T}(t)\right]$. This proof is completed.

\section{Distributed Fusion Estimator}

In the preceding sections, we have obtained the local estimators at the state update points and their covariance matrices. Applying the distributed matrix weighted optimal fusion estimation algorithm in the linear minimum variance sense [8], we can obtain the distributed fusion estimator as follows:

$$
\widehat{x}_{o}(t)=\sum_{i=1}^{L} A_{i}(t) \widehat{x}_{i}(t) .
$$

The optimal weighted matrices are computed by

$$
\left[A_{1}(t), \ldots, A_{L}(t)\right]=\left(e^{T} P^{-1}(t) e\right)^{-1} e^{T} P^{-1}(t),
$$




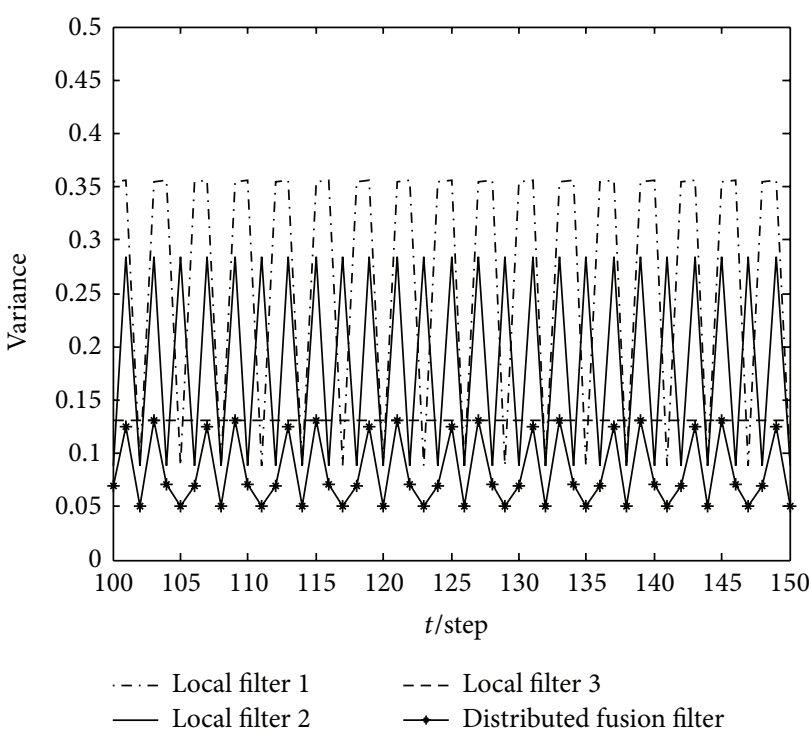

(a) The first state component

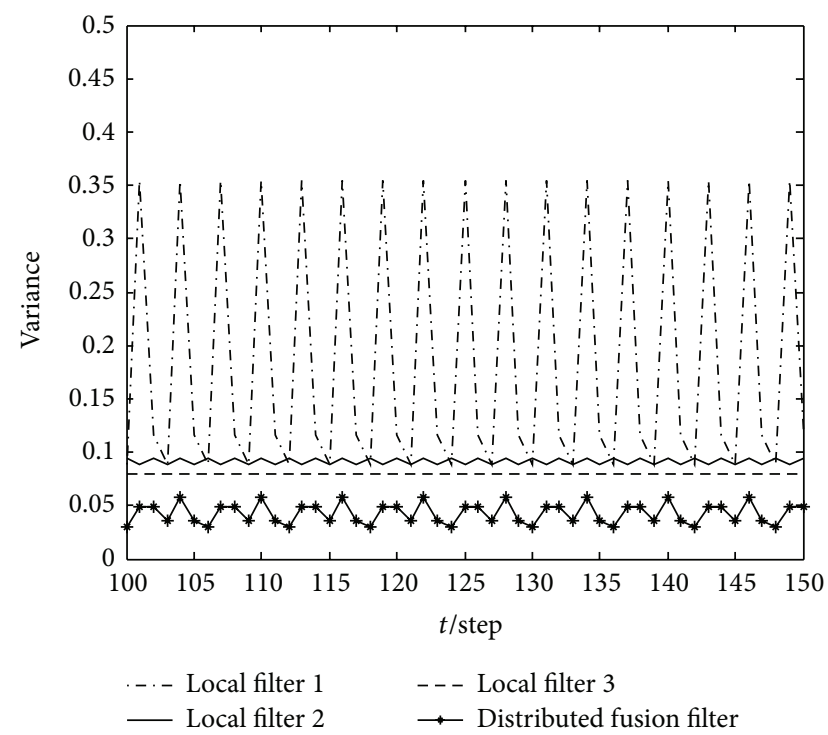

(b) The second state component

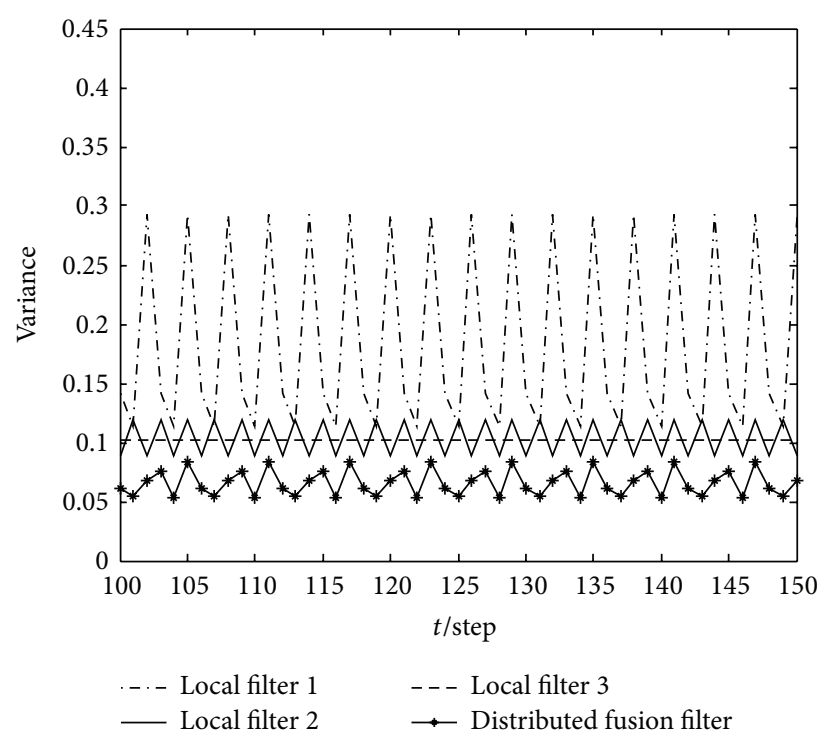

(c) The third state component

FIGURE 3: Comparison of variances of distributed fusion filter and local filters.

where $P(t)=\left(P_{i j}(t)\right)$ is an $n L \times n L$ matrix whose $(i, j)$ block is $P_{i j}(t)$ and $e=\left[I_{n}, \ldots, I_{n}\right]^{T}$ is an $n L \times n$ matrix. Then, the optimal fusion estimation error variance matrix is computed by

$$
P_{o}(t)=\left(e^{T} P^{-1}(t) e\right)^{-1}
$$

Furthermore, we have $P_{o}(t) \leq P_{i}(t)$.

Remark 6. Compared to the centralized fusion estimator, the distributed fusion estimator has the flexibility, fault tolerance, and reliability since it has the distributed parallel structure [8].

\section{Simulation}

An uninterruptible power system (UPS) with three sensors subject to the multiplicative noises is taken as an example to demonstrate the effectiveness and applicability of the proposed method. We consider the UPS with 1KVA. The discrete-time model (1) can be obtained with sampling time $10 \mathrm{~ms}$ at half-load operating point as follows [20]:

$$
\begin{aligned}
x(t+1) & \\
& =\left(\begin{array}{ccc}
0.9226 & -0.6330 & 0 \\
1 & 0 & 0 \\
0 & 1 & 0
\end{array}\right) x(t)+\left(\begin{array}{c}
0.5 \\
0 \\
0.2
\end{array}\right) w(t)
\end{aligned}
$$



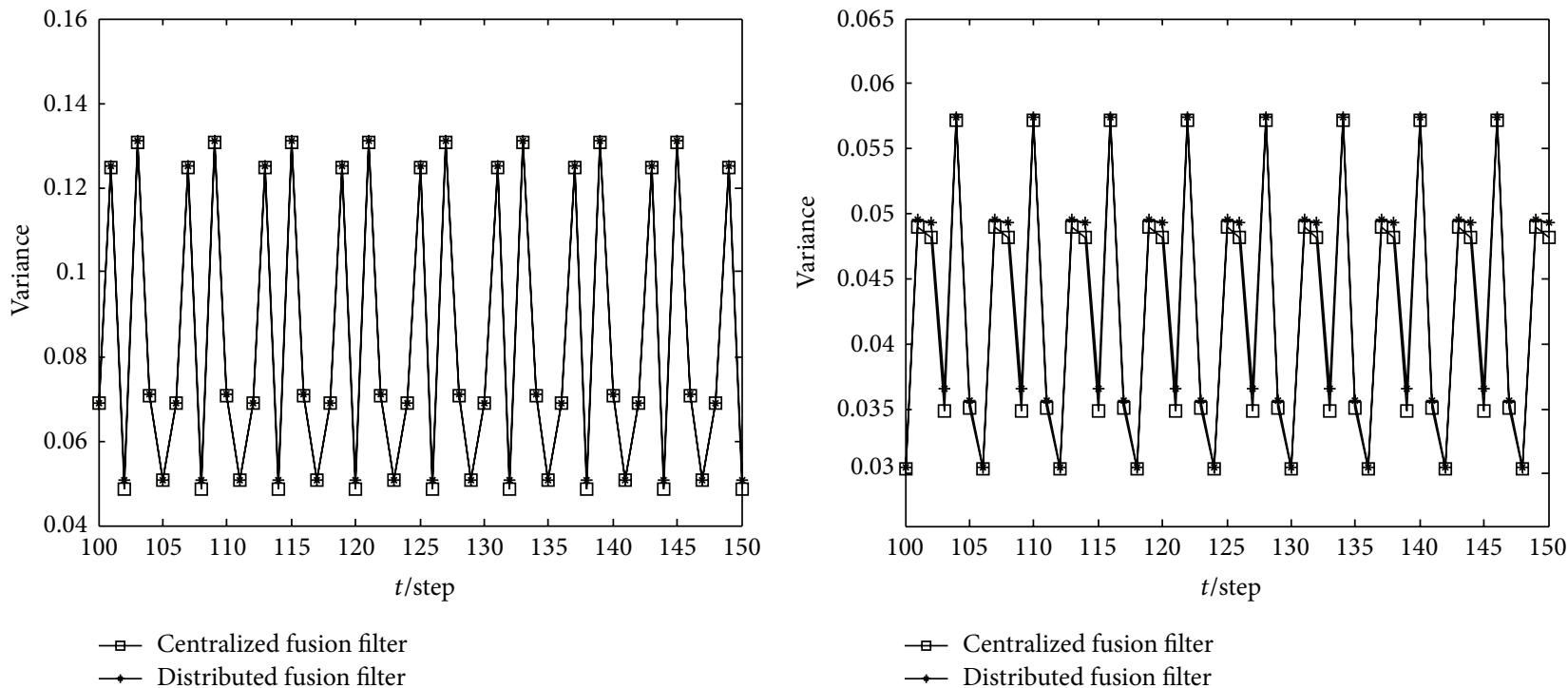

$\rightarrow$ Centralized fusion filter

$\rightarrow$ Distributed fusion filter

(a) The first state component

(b) The second state component

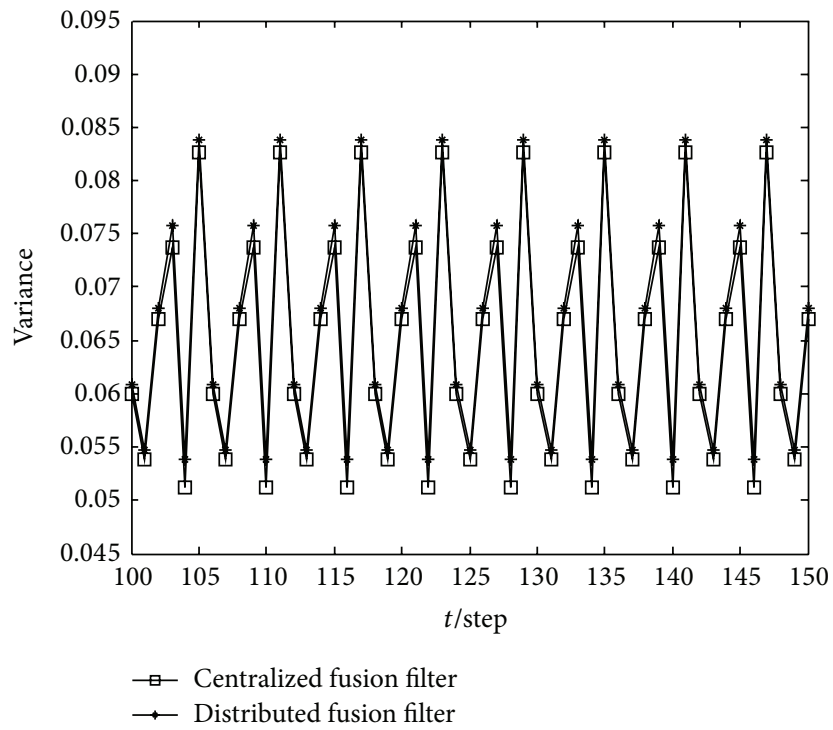

(c) The third state component

FIGURE 4: Comparison of variances of centralized and distributed fusion filters.

$$
\begin{array}{r}
\left.y_{i}\left(l_{i} t\right)=\left(\begin{array}{lll}
(23.738 & 20.287 & 0
\end{array}\right)+\xi_{i}\left(l_{i} t\right) H_{1 i}\right) x_{i}\left(l_{i} t\right)+v_{i}\left(l_{i} t\right), \\
i=1,2,3,
\end{array}
$$

where $w(t), v_{i}\left(l_{i} t\right)$, and $\xi_{i}\left(l_{i} t\right)$ are uncorrelated white noises with zero mean and variances $Q_{w}, Q_{v_{i}}$, and $Q_{\xi_{i}}$. In simulation, we take $Q_{w}=0.64, Q_{\xi_{1}}=1.2, Q_{\xi_{2}}=0.7, Q_{\xi_{3}}=0.3$, $Q_{v_{1}}=3, Q_{v_{2}}=2, Q_{v_{3}}=1, l_{1}=1, l_{2}=2, l_{3}=3, H_{11}=$ $\left[\begin{array}{lll}8 & 12 & 6\end{array}\right], H_{12}=\left[\begin{array}{lll}10 & 8 & 5\end{array}\right], H_{13}=\left[\begin{array}{lll}8 & 6 & 10\end{array}\right]$, the initial values $x(0)=0$, and $P_{0}=0.1 I_{3}$. The sampling case is shown in Figure 1. Figure 2 gives the distributed fusion estimator. We see that the fusion estimator has the effective estimation performance. Figure 3 gives the comparison of variances of the distributed fusion estimator and local estimators. We see that the proposed fusion estimator outperforms the local estimators. Figure 4 gives the comparison of variances of the distributed fusion estimator and centralized fusion estimator. We see that the distributed fusion estimator has the small accuracy loss. However, it is significant that the distributed fusion estimator has better reliability than the centralized fusion estimator since it is convenient to detect and isolate the faults of sensors from distributed structure.

\section{Conclusion}

A distributed fusion estimator has been designed for systems with multiple sensors of different sampling rates and observation multiplicative noises. Compared with the centralized 
fusion estimator, it has the small accuracy loss and better reliability. Sampling period of each sensor is uniform and the integer multiple of the state update period. By establishing the state space model at the observation sampling points, the local filters at the observation sampling points are obtained. Further, the local estimators at the state update points are obtained by the filtering and prediction approach. They avoid the state augmentation and have a good real-time property. The cross-covariance matrices between any two local estimators are derived. The distributed fusion estimator is obtained by well-known weighted fusion estimation algorithm in linear minimum variance sense.

\section{Conflict of Interests}

The authors declare that there is no conflict of interests regarding the publication of this paper.

\section{Acknowledgments}

This work is supported by the Natural Science Foundation of China (NSFC-60874062 and NSFC-61174139), by the Program for New Century Excellent Talents in University (no. NCET-10-0133), by the Chang Jiang Scholar Candidates Program for Provincial Universities in Heilongjiang (no. 2013CJHB005), by Science and Technology Innovative Research Team in Higher Educational Institutions of Heilongjiang Province (No. 2012TD007), by the Program for High-qualified Talents (no. Hdtd2010-03), and by the Electronic Engineering Provincial Key Laboratory.

\section{References}

[1] N. Nahi, "Optimal recursive estimation with uncertain observation," IEEE Transactions on Information Theory, vol. 15, no. 4, pp. 457-462, 1969.

[2] W. L. de Koning, "Optimal estimation of linear discrete-time systems with stochastic parameters," Automatica, vol. 20, no. 1, pp. 113-115, 1984.

[3] F. Carravetta, A. Germani, and M. Raimondi, "Polynomial filtering of discrete-time stochastic linear systems with multiplicative state noise," IEEE Transactions on Automatic Control, vol. 42, no. 8, pp. 1106-1126, 1997.

[4] M. Basin, J. Pérez, and M. Skliar, "Optimal filtering for polynomial system states with polynomial multiplicative noise," International Journal of Robust and Nonlinear Control, vol. 16, no. 6, pp. 303-314, 2006.

[5] M. V. Basin and D. Calderón Álvarez, "Optimal filtering for incompletely measured polynomial systems with multiplicative noise," Circuits, Systems, and Signal Processing, vol. 28, no. 2, pp. 223-239, 2009.

[6] J. Ma and S. L. Sun, "Optimal linear estimation for systems with multiplicative noise uncertainties and multiple packet dropouts," IET Signal Processing, vol. 6, no. 9, pp. 839-848, 2012.

[7] Z. L. Deng, Information Fusion Filtering Theory with Applications, Harbin Institute of Technology Press, Harbin, China, 2007.

[8] S. L. Sun and Z. L. Deng, "Multi-sensor optimal information fusion Kalman filter," Automatica, vol. 40, no. 6, pp. 1017-1023, 2004.
[9] Z. L. Deng, Y. Gao, C. B. Li, and G. Hao, "Self-tuning decoupled information fusion Wiener state component filters and their convergence," Automatica, vol. 44, no. 3, pp. 685-695, 2008.

[10] C. L. Wen, B. Lu, and Q. B. Ge, "Data fusion algorithm based on filtering step by step," Acta Electronica Sinica, vol. 32, no. 8, pp. 1264-1267, 2004.

[11] Q. B. Ge, G. A. Wang, T. H. Tang, and C. Wen, "The research on asynchronous data fusion algorithm based on sampling of rational number times," Acta Electronica Sinica, vol. 34, no. 3, pp. 543-548, 2006.

[12] C. L. Wen, Z. G. Chen, L. P. Yan, and D. Zhou, "Multiscale recursive fusion estimation based on dynamic systems of multirate sensors," Journal of Electronics and Information Technology, vol. 25, no. 3, pp. 306-312, 2003.

[13] L. P. Yan, B. S. Liu, and D. H. Zhou, "The modeling and estimation of asynchronous multirate multisensor dynamic systems," Aerospace Science and Technology, vol. 10, no. 1, pp. 63-71, 2006.

[14] C. Y. Xiao, J. Ma, and S. L. Sun, "Design of information fusion filter for a class of multi-sensor asynchronous sampling systems," in Proceedings of the Chinese Control and Decision Conference (CCDC '11), pp. 1081-1084, May 2011.

[15] L. P. Yan, B. S. Liu, and D. H. Zhou, "Asynchronous multirate multisensor information fusion algorithm," IEEE Transactions on Aerospace and Electronic Systems, vol. 43, no. 3, pp. 1135-1146, 2007.

[16] L. P. Yan, D. H. Zhou, M. Y. Fu, and Y. Q. Xia, "State estimation for asynchronous multirate multisensor dynamic systems with missing measurements," IET Signal Processing, vol. 4, no. 6, pp. 728-739, 2010.

[17] H. L. Lin, J. Ma, and S. L. Sun, "Optimal state filters for a class of non-uniform sampling systems," Journal of Systems Science and Mathematics, vol. 32, no. 6, pp. 768-779, 2012.

[18] Z. H. Deng, L. P. Yan, and M. Y. Fu, "Multirate multisensor data fusion based on missing measurements," Systems Engineering and Electronics, vol. 32, no. 5, pp. 886-958, 2010.

[19] Y. L. Liu, L. P. Yan, and Y. Q. Xia, "Multirate multisensor distributed data fusion algorithm for state estimation with cross-correlated noises," in Proceedings of the 32nd Chinese Control Conference, Technical Committee on Control Theory, Chinese Association of Automation, Xi'an, China, July 2013.

[20] Y. Rong, The design of iterative learning control and its application to UPS [M.S. thesis], Fuzhou University, Fuzhou, China, 2003. 


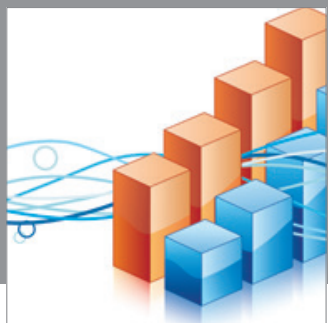

Advances in

Operations Research

mansans

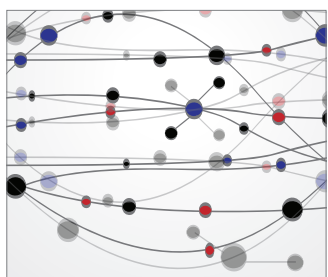

The Scientific World Journal
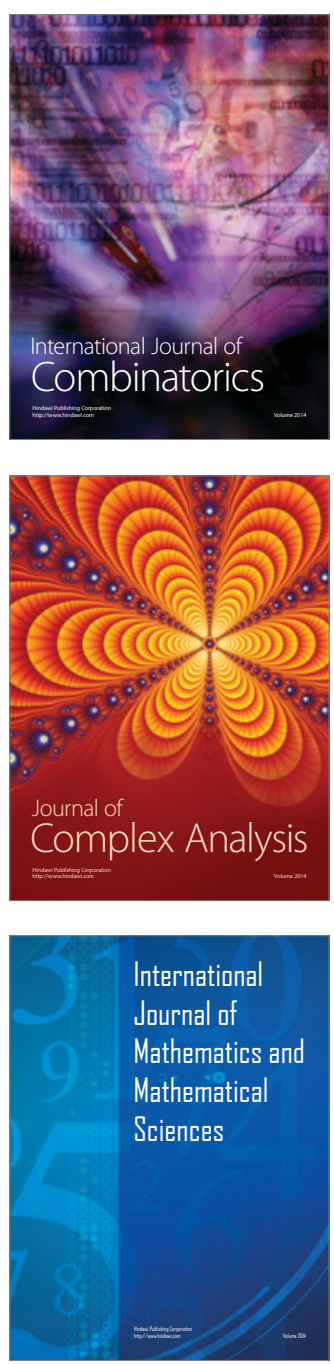
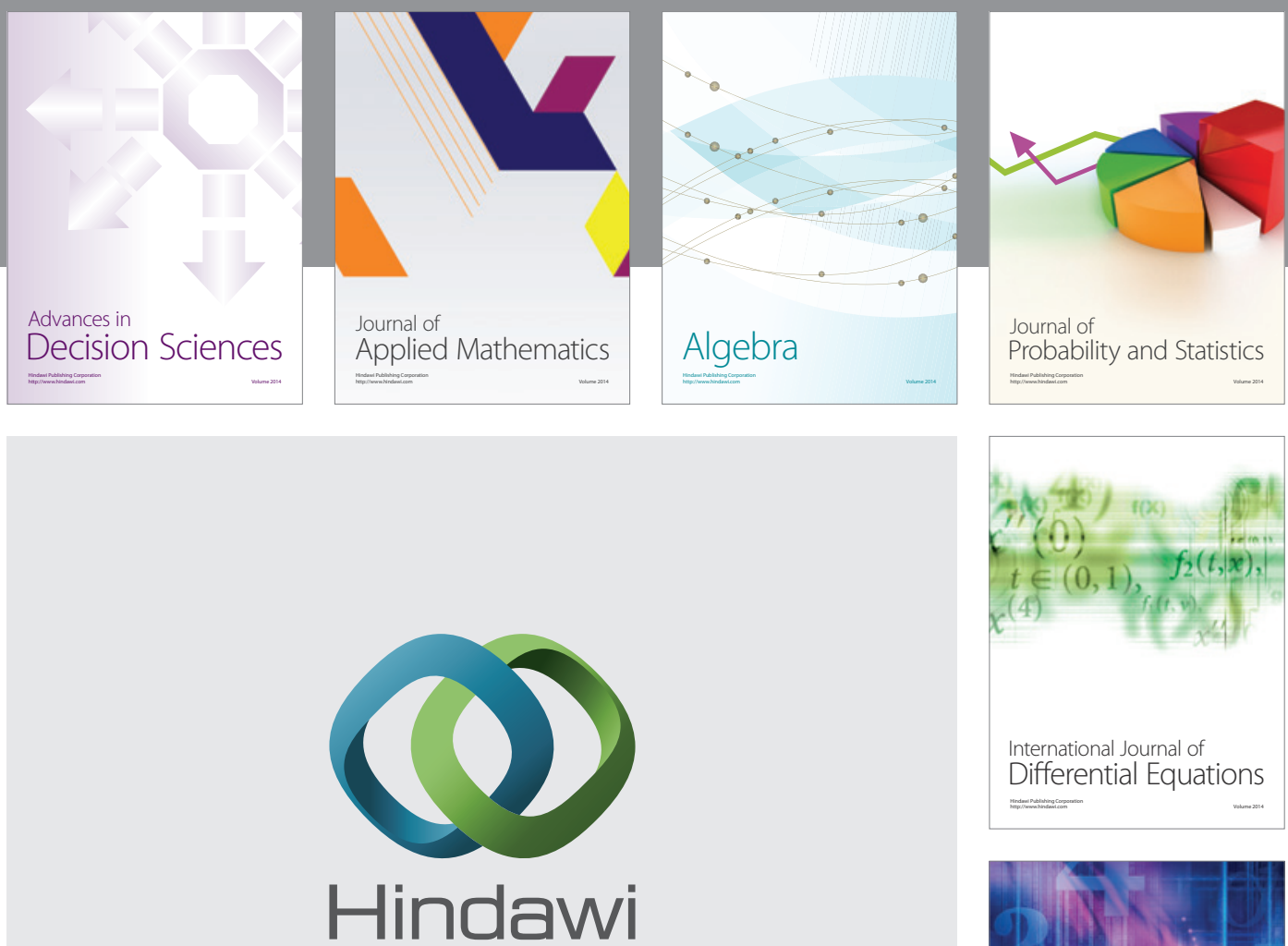

Submit your manuscripts at http://www.hindawi.com
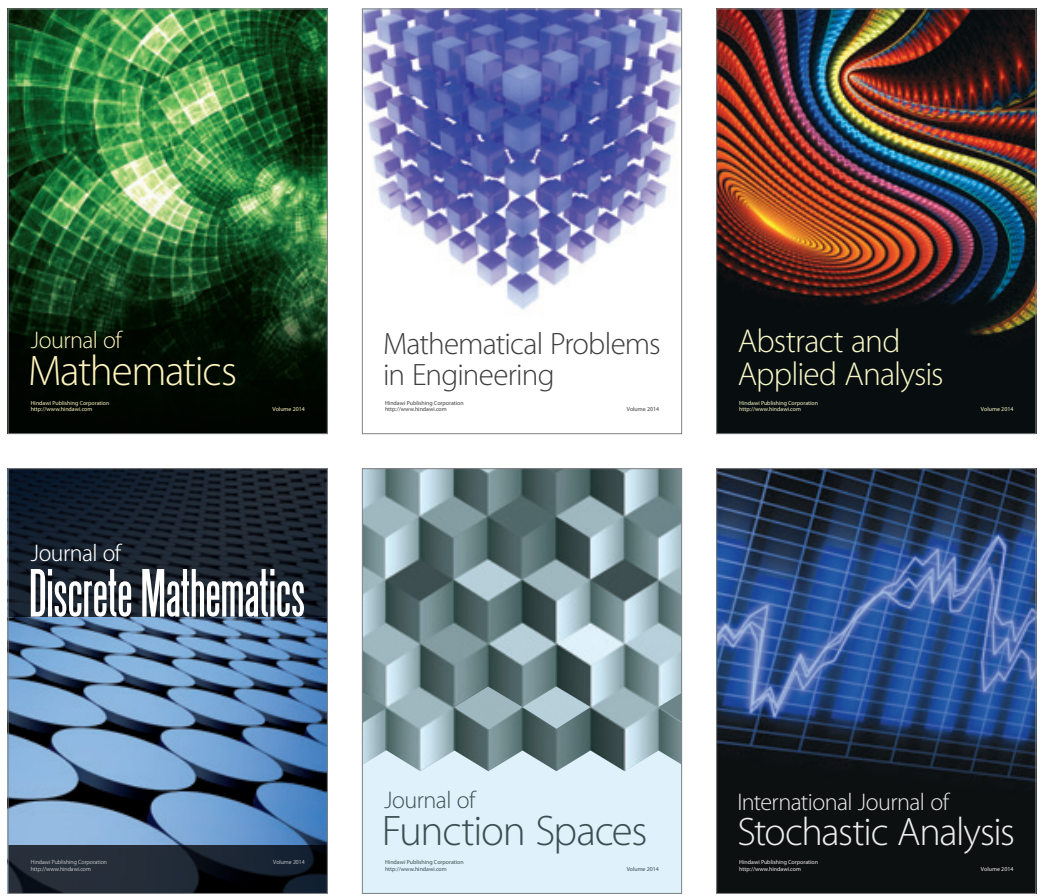

Journal of

Function Spaces

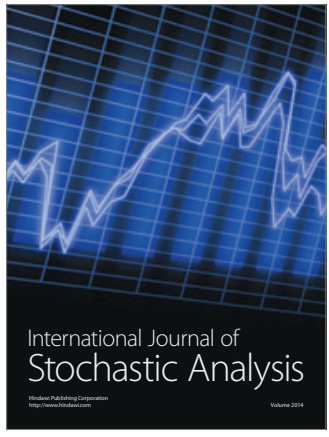

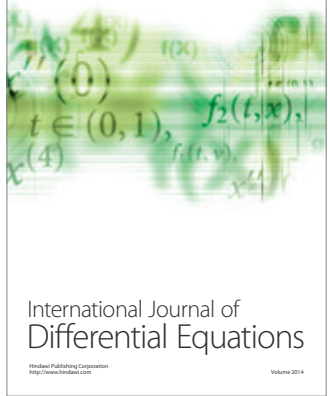
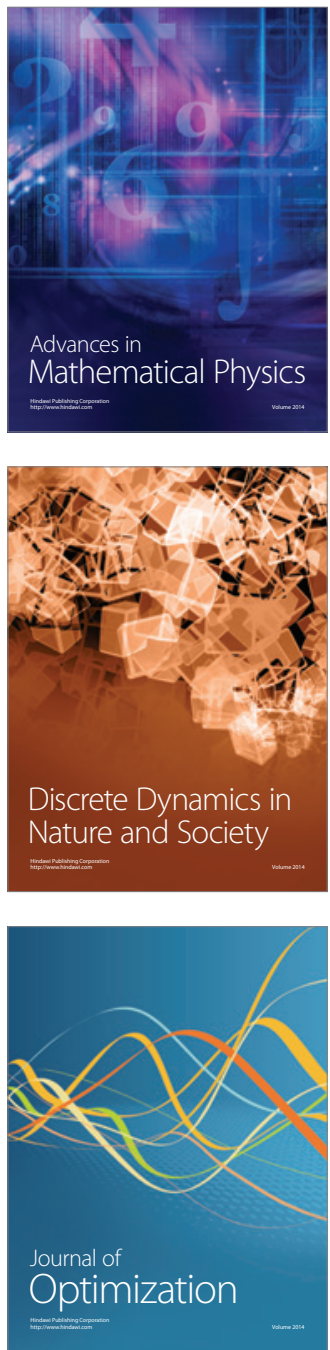